\title{
Distributed Computing for the Factory-Floor: a Real-Time Approach using WorldFIP Networks
}

\author{
Eduardo Tovar* \\ Department of Computer Engineering, Polytechnic Institute of Porto, Portugal \\ Francisco Vasques \\ Department of Mechanical Engineering, University of Porto, Portugal
}

\begin{abstract}
Field communication systems (fieldbuses) are widely used as the communication support for distributed computer-controlled systems (DCCS) within all sort of process control and manufacturing applications. There are several advantages in the use of fieldbuses as a replacement for the traditional point-to-point links between sensors/actuators and computer-based control systems, within which the most relevant is the decentralisation and distribution of the processing power over the field. A widely-used fieldbus is the WorldFIP, which is normalised as European standard EN 50170.

Using WorldFIP to support DCCS, an important issue is "how to guarantee the timing requirements of the real-time traffic"? WorldFIP has very interesting mechanisms to schedule data transfers, since it explicitly distinguishes periodic and aperiodic traffic. In this paper we describe how WorldFIP handles these two types of traffic, and more importantly, we provide a comprehensive analysis on how to guarantee the timing requirements of the real-time traffic.
\end{abstract}

Keywords : Factory-floor communications; Hard real-time systems; WorldFIP Networks; Distributed computer-controlled systems. 


\section{Introduction}

In the past decade manufacturing schemes have changed dramatically. In particular, the Computer Integrated Manufacturing [1] concept has been stressed as a means to achieve greater production competitiveness [2]. The driving forces behind the changes also resulted from the increased development and utilisation of new technologies that make massive use of microprocessor-based equipment. Integration implies that different subsystems of the manufacturing environment interact and co-operate with each other. This means transfer, storage and processing of information in a widespread environment. In other words, integration requires efficient support for data communications.

Nowadays, communication networks are available to virtually every aspect of the manufacturing environment, ranging from the production planning to the field level [3]. However, the use of communication networks at the field level is a much more recent trend [4]. Indeed, only more recently network interfaces become cost-effective for the interconnection of devices such as sensors and actuators, which, in the majority of the cases, are expected to be cheaper than the equipment typically interconnected at upper control levels of the manufacturing environment (e.g., workstations and numerically -controlled machines).

The field level includes process-relevant field devices, such as sensors and actuators. The process control level is hierarchically located above the field level, and directly influences it in the form of control signals. If the control is computer-based, the process control level uses data received from sensors to compute new commands, which are then transmitted to the actuators.

A computer-controlled system can have a centralised architecture. By centralised architecture we mean that there is only one single computer system unit, which has I/O capabilities to support both the instrumentation (sensors/actuators) and the man-machine interfaces. The sensors and actuators are connected to the computer system via point-to-point links. Fig. 1a) illustrates such kind of architecture.

There are however several advantages in using a field level communication network as the replacement for the point-to-point links between the sensors/actuators and the computer system. As it can be depicted from Fig. 1b), a cost reduction can be obtained by replacing a significant part of the wiring by a field level communication network. Naturally, the use of a single wire brings other important advantages, such as easier installation and maintenance, easier detection and localisation of cable faults, and easier expansion due to the modular nature of the network.

With the increased availability of low cost technology, the decentralised computer-controlled architecture can easily evolve to a distributed computer-controlled architecture. Basically, the difference relies on the distribution of the control algorithms. In a centralised computer-controlled architecture, all the control algorithms are implemented in a single computer system. In a decentralised computer-controlled architecture, the control algorithms are also centralised in a single computer system (now also a network node), even if some processing tasks (signal conditioning or pre-processing operations) may be executed in the network nodes that interface to the sensors and actuators. Contrarily, in a distributed computer-controlled architecture, the tasks of the control algorithms may be distributed throughout several computing nodes. Fig. 2 depicts the organisation of a distributed computer-controlled architecture. 
a)
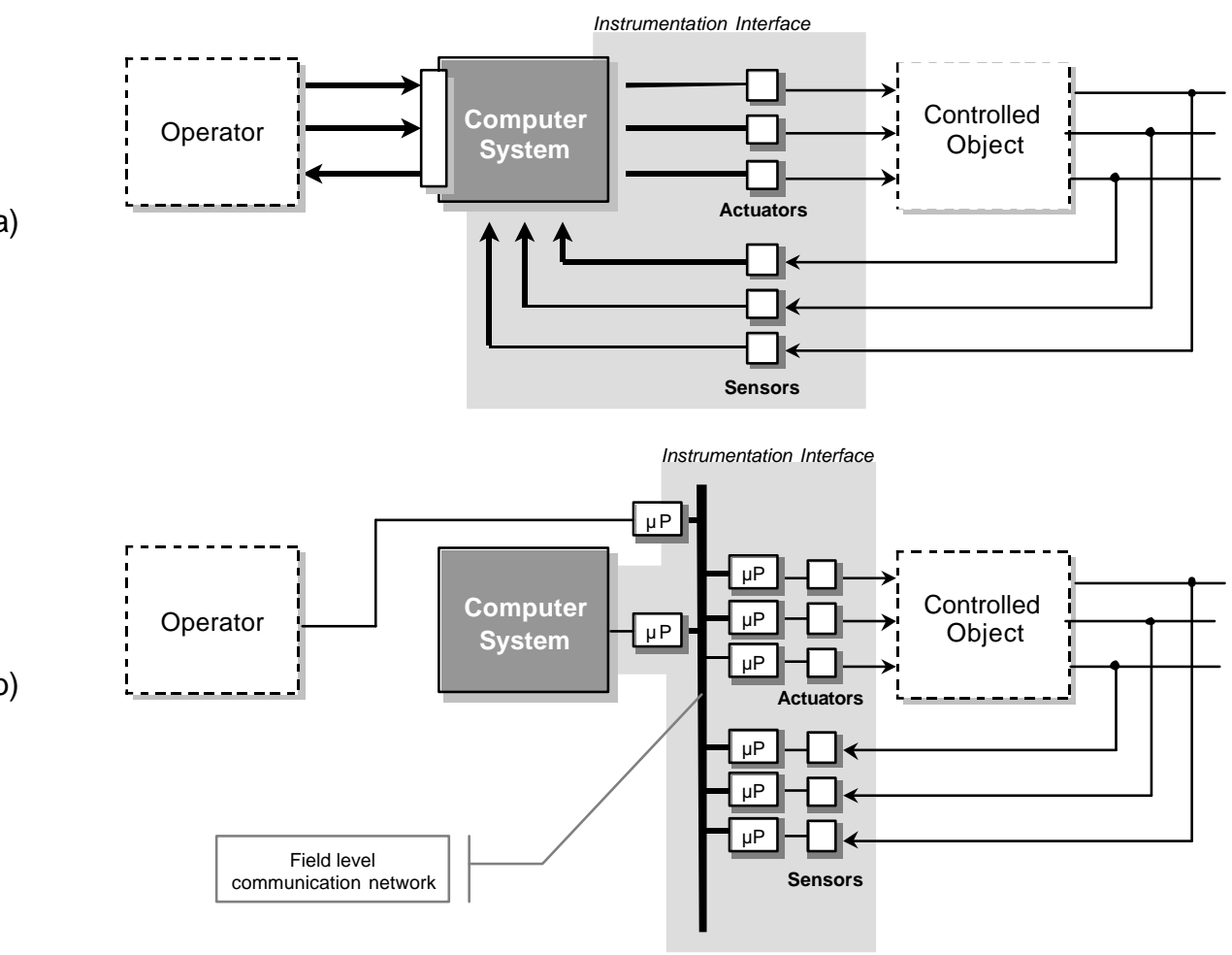

Fig. 1. This figure highlights the main advantage of a decentralised computer-controlled architecture (b) when compared to a centralised computer-controlled architecture (a)

The ability to support distributed control algorithms is another advantage of using a field level network. This eases the design of computer-controlled systems where decentralisation of control and measurement tasks, as well as the number of microprocessor-controlled devices is ceaselessly increasing.

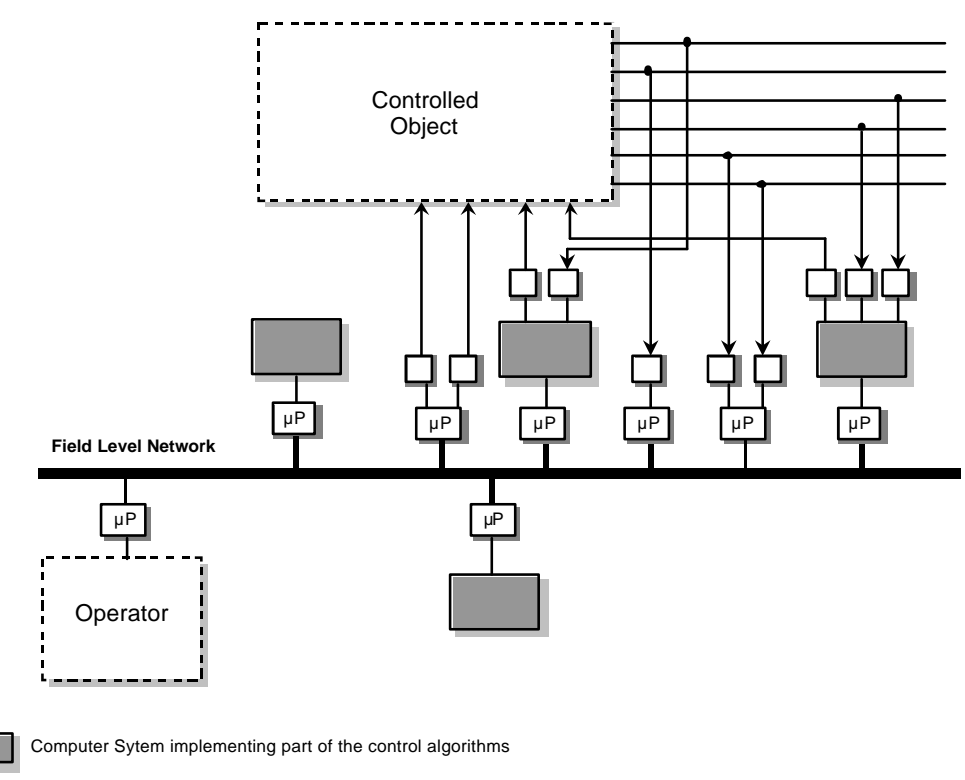

Fig. 2 This figure represents a distributed computer-controlled architecture

Field level networks aimed at the interconnection of sensors, actuators and controllers are commonly known as fieldbus networks. In the past, the fieldbus scope was dominated by vendor specific solutions, which were mostly restricted to specific application areas. Moreover, concepts behind each proposed network were highly dependent on the manufacturer of the automation system, each one with different technical implementations and also claiming to fulfil different application requirements, or the same requirements with different technical 
solutions [5]. More recently, standardised fieldbuses supporting the open system concept, thus vendor independent, started to be commonly used. Particular relevance must be given to the European Standard EN 50170 [6], which encompasses three widely used fieldbuses: P-NET [7], PROFIBUS [8] and WorldFIP [9]. All these fieldbus protocols aim at the support of distributed computer-controlled systems (DCCS).

This paper addresses the ability of WorldFIP to cope with the real-time requirements of DCCS. Typically, DCCS include process variables that must be transferred between network devices, either in a periodic or in an aperiodic basis. The WorldFIP protocol is designed to support both types of traffic. A potential large leap towards its use in DCCS relies on the evaluation of its temporal behaviour. Particularly, an important problem associated with the WorldFIP fieldbus is its inability to guarantee the timing requirements associated to the aperiodic traffic. As it will be seen, realtime requirements for the periodic traffic can be easily guaranteed by the WorldFIP protocol. However, for the aperiodic traffic more complex analysis must be made to evaluate the message's response time, in order to guarantee its real-time requirements. The main focus of this paper will be in the analysis of the aperiodic message's response time.

The remainder of this paper is organised as follows. Section 2 describes the most important concepts of the WorldFIP networks. In Section 3 we evaluate the worst-case response time for the aperiodic traffic as a function of the periodic traffic schedule, thus performing an integrated analysis of both types of traffic. In Section 4 we present a numerical example, which highlights the relevance of the proposed methodology. Finally, in Section 5 some conclusions are drawn.

\section{Basic Analysis of the WorldFIP protocol}

A WorldFIP network interconnects stations with two types of functionality: bus arbitration and production/consumption functions. At any given instant, only one station can perform the function of active bus arbitration. Hence, in WorldFIP, the medium access control (MAC) is centralised, and performed by the active bus arbitrator (BA).

WorldFIP supports two basic types of transmission services: exchange of identified variables and exchange of messages. In this paper we address WorldFIP networks supporting only the exchange of identified variables, since they are the basis of WorldFIP realtime services. The exchange of messages, which is used to support manufacturing message services (MMS) [10], is out of the scope of this paper.

\subsection{Producer/Distributor/Consumer Concept}

In WorldFIP, the exchange of identified variables is based on a producer/distributor/consumer (PDC) model, which relates producers and consumers within the distributed system. In this model, for each process variable there is one, and only one producer, and one or more consumers. For instance, consider the variable associated with a process sensor. The station that provides the value of the variable will act as the producer of the variable and its value will be provided to all the consumers of the variable (e.g., the station that acts as process controller for that variable or the station that is responsible for building an historical data base).

In order to manage transactions associated with a single variable, a unique identifier is associated with each variable. The WorldFIP data link layer (DLL) is made up of a set of produced and consumed buffers, which can be locally accessed (through application layer (AL) services) or remotely accessed (through network services). 
The AL provides two basic services to access the DLL buffers: L_PUT.req, to write a value in a local produced buffer, and L_GET. req to obtain a value from the local consumed buffer. None of these AL services generate activity on the bus.

Produced and consumed buffers can be also remotely accessed through a network transfer (service also known as buffer transfer). The bus arbitrator broadcasts a question frame ID_DAT, which includes the identifier of a specific variable. The DLL of the station that has the corresponding produced buffer, responds with the value of the variable, using a response frame RP_DAT. The DLL of the station that contains the produced buffer then sends an indication of transmission of the value to the AL ( L_SENT. ind). The DLL of the station(s) that has the consumed buffers accepts the value contained in the RP_DAT, overwriting the previous value and notifying the local AL with a L_RECEIVED. ind.

\subsection{Buffer Transfer Timings}

A buffer transfer implies the transmission of a pair of frames: ID_DAT, followed by RP_DAT. We denote this sequence as an elementary transaction. The duration of this transaction equals the time needed to transmit the $I D \_D A T$ frame, added to the time needed to transmit the RP_DAT frame, and finally added to twice the turnaround time $\left(t_{r}\right)$. The turnaround time is the time elapsed between any two consecutive frames.

Every transmitted frame is encapsulated with control information from the physical layer (PL). Specifically, an ID_DAT frame has always 8 bytes (corresponding to a 2-bytes identifier plus 6 bytes of control information), whereas a RP_DAT frame has also 6 bytes of control information plus up to 128 bytes of useful data. The duration of a message transaction is:

$$
C=\frac{\text { len }\left(\mathrm{id} \_\mathrm{dat}\right)+\operatorname{len}\left(\mathrm{rp} \_\mathrm{dat}\right)}{b p s}+2 \times t_{r}
$$

where $b p s$ stands for the network data rate and len $(<f$ rame $>)$ is the length, in bits, of frame $<$ frame $>$.

For instance, assuming a variable with a 4-bytes data field, if $t_{r}=20 \mu \mathrm{s}^{1}$ the network data rate is $2.5 \mathrm{Mbps}$ then, the duration of an elementary transaction will be $(64+80) / 2.5+2 \times 20=97.6 \mu \mathrm{s}$ (equation (1)).

\subsection{Bus Arbitrator Table}

In WorldFIP networks, the bus arbitrator table (BAT) regulates the scheduling of all buffer transfers. The BAT imposes the timings of the periodic buffer transfers, and also regulates the aperiodic buffer transfers, as it will be explained in Section 2.4.

Assume a distributed system within which 6 variables are to be periodically scanned, with scan rates as shown in Table 1. The WorldFIP BAT must cope with these real-time requirements.

Table 1: Example set of periodic buffer transfers

\begin{tabular}{l|cccccc} 
Identifier & A & B & C & D & E & F \\
\hline Periodicity (ms) & 1 & 2 & 3 & 4 & 4 & 6
\end{tabular}

\footnotetext{
${ }^{1}$ The turnaround time $\left(t_{r}\right)$ is imposed [9] to be within the interval $10 \times(1 / b p s) \leq t_{r} \leq 70 \times(1 / b p s)$.
} 
Two important parameters are associated with a WorldFIP BAT: the micro-cycle (elementary cycle) and the macro-cycle. The micro-cycle imposes the maximum rate at which the BA performs a set of scans. Usually, the micro-cycle is set equal to highest common factor (HCF) of the required scan periodicities. Using this HCF rule, and for the example of Table 1 , the value for the micro-cycle is set to $1 \mathrm{~ms}$. A possible schedule for all the periodic scans is illustrated in Fig. 3, where we consider $C=97.6 \mu \mathrm{s}$ (1) for each elementary transaction.

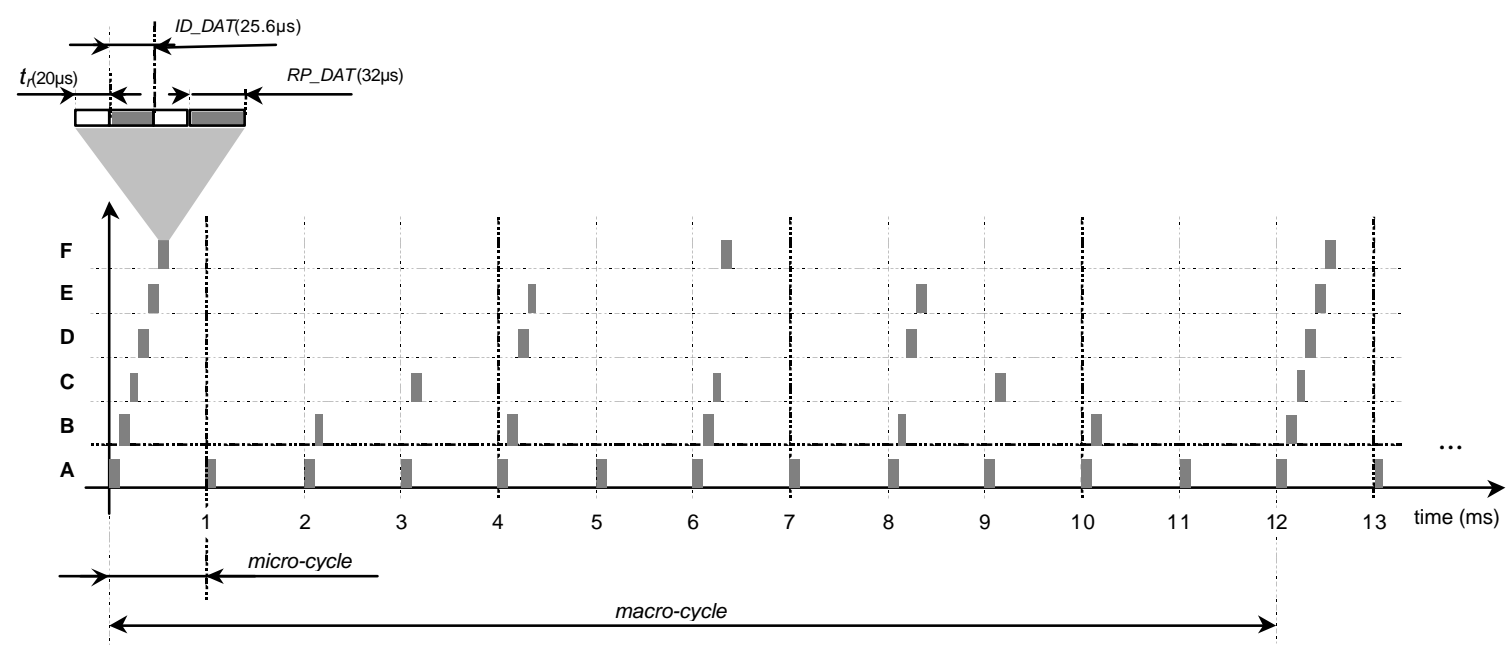

Fig. 3 This figure illustrates a possible schedule for the periodic buffer transfers of Table 1

It is easy to depict that, for this example, the sequence of micro-cycles is repeated after each 12 micro-cycles. This sequence of micro-cycles is said to be a macro-cycle, and its length is given by the lowest common multiple (LCM) of the scan periodicities. The HCF/LCM approach for building the WorldFIP BAT has the following properties:

1. The scanning periods of the variables are multiples of the micro-cycle;

2. The variables are not scanned at exactly regular intervals. For the given example, only variables $A$ and $B$ are scanned exactly in the same "slot" within the micro-cycle. All other variables suffer from a slight communication jitter. For instance, concerning variable $F$, the interval between micro-cycles 1 and 7 is $(1-5 \times 0.098)+5+(3 \times 0.098)=5.80 \mathrm{~ms}$, whereas the interval between micro-cycles 7 and 13 is $(1-$ $3 \times 0.098)+5+(5 \times 0.098)=6.20 \mathrm{~ms}$.

3. The length of the macro-cycle can induce a memory size problem, since the table parameters must be stored in the BA. For instance, if scanning periodicities of variables $E$ and $F$ were, respectively, $5 \mathrm{~ms}$ and $7 \mathrm{~ms}$, the length of the macro-cycle would be 420 micro-cycles instead of only 12.

Both the communication jitter and memory size problems have been addressed in the literature. In [11] the authors discuss different methodologies for reducing the BAT size, without penalising the communication jitter. The idea is very simple, and it basically consists on reducing some of the scan periodicities in order to obtain a harmonic pattern. The problem of table size has also been addressed in [12,13], however in a different perspective. In the referred work, the authors discuss an online scheduler (instead of storing the schedule in the BA's memory), which is not directly applicable to the WorldFIP case.

It is also worth mentioning that the schedule shown in Fig. 3 represents a macro-cycle composed of synchronous micro-cycles, that is, for the specific example, each micro-cycle starts exactly $1 \mathrm{~ms}$ after the previous one. Within a micro-cycle, the spare time between the end of the last scan for a periodic variable and the end of the micro-cycle can be used by the BA to process aperiodic requests for buffer transfers (see Section 2.4), message transfers and padding identifiers. A WorldFIP BA can also manage asynchronous micro-cycles, 
not transmitting padding identifiers at the end of the micro-cycle. In such case, a new micro-cycle starts as soon as the periodic traffic is performed and there are no pending aperiodic buffer transfers or message transfers. Initial periodicities are not respected, since identifiers may be more frequently scanned.

\subsection{Aperiodic Buffer Transfers}

In a WorldFIP system, not all the variables are to be included in the BAT. Some may only be occasionally exchanged, and thus do not need to be periodically scanned. Typically such exchanges will concern application events or alarms, which by their own nature do not occur with a periodic pattern. Therefore, it is preferable to map these variables into aperiodic buffer transfers, in order to reduce the network load.

The BA handles the aperiodic buffer transfers after processing the periodic traffic in a micro-cycle. The portion of the micro-cycle reserved for the periodic buffer exchanges is denoted as the periodic window, whereas the time left after the periodic window is denoted as the aperiodic window. The aperiodic buffer transfers take place in three stages (Fig. 4):

1. A station with a pending aperiodic request must wait for its next periodic buffer transfer (say periodic variable $X$ ) to notify the $\mathrm{BA}$, setting an aperiodic request bit in the RP_DAT frame. The bus arbitrator stores the indication of a yet not identified aperiodic request in a queue of requests for variable transfers. At the end of this interval, the BA is aware of a pending request in the station that produces periodic variable $X$.

2. In a subsequent aperiodic window, the BA asks the producer of the variable $X$ ( $I D \_R Q$ frame) to transmit the list of its pending aperiodic requests. The producer of $X$ responds with a $R P \_R Q$ (list of identifiers) frame. This list is placed in another BA's queue, the ongoing aperiodic queue. At the end of this interval the BA knows which are the requested identifiers.

3. Finally, the BA processes requests for aperiodic transfers that are stored in its ongoing aperiodic queue. For each transfer, the BA uses the same mechanism as that used for the periodic buffer transfers (ID_DAT followed by RP_DAT).

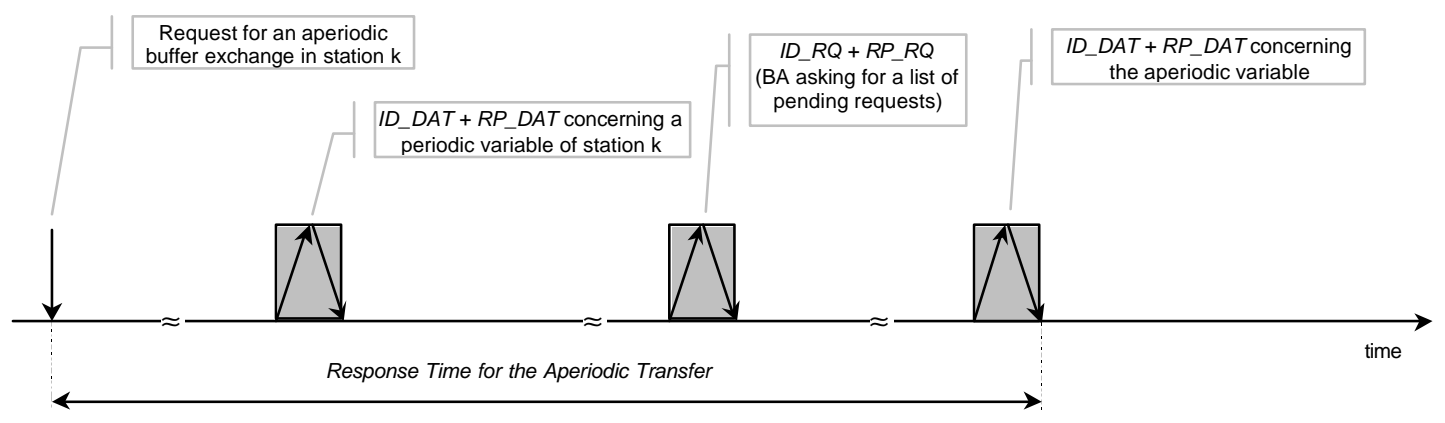

Fig. 4. Timings for transactions associated with the processing of one aperiodic variable

It is important to note that a station can only request aperiodic transfers using responses to periodic variables that it produces and which are configured in the BAT. Finally, it is important to stress that the urgent queue in the BA is only processed if, and only if, the BA's ongoing aperiodic queue is empty. As it can be depicted from Fig. 5, traffic concerning the aperiodic buffer transfers (transactions ID_RQ/RP_RQ or ID_DAT/RP_DAT) can only be carried out if there is time left in a specific micro-cycle to completely process them.

As previously mentioned, in this paper we only consider the transfer of identified variables, for both periodic and aperiodic buffer transfers. Concerning the aperiodic requests, we consider that all these requests use the Urgent priority level. 


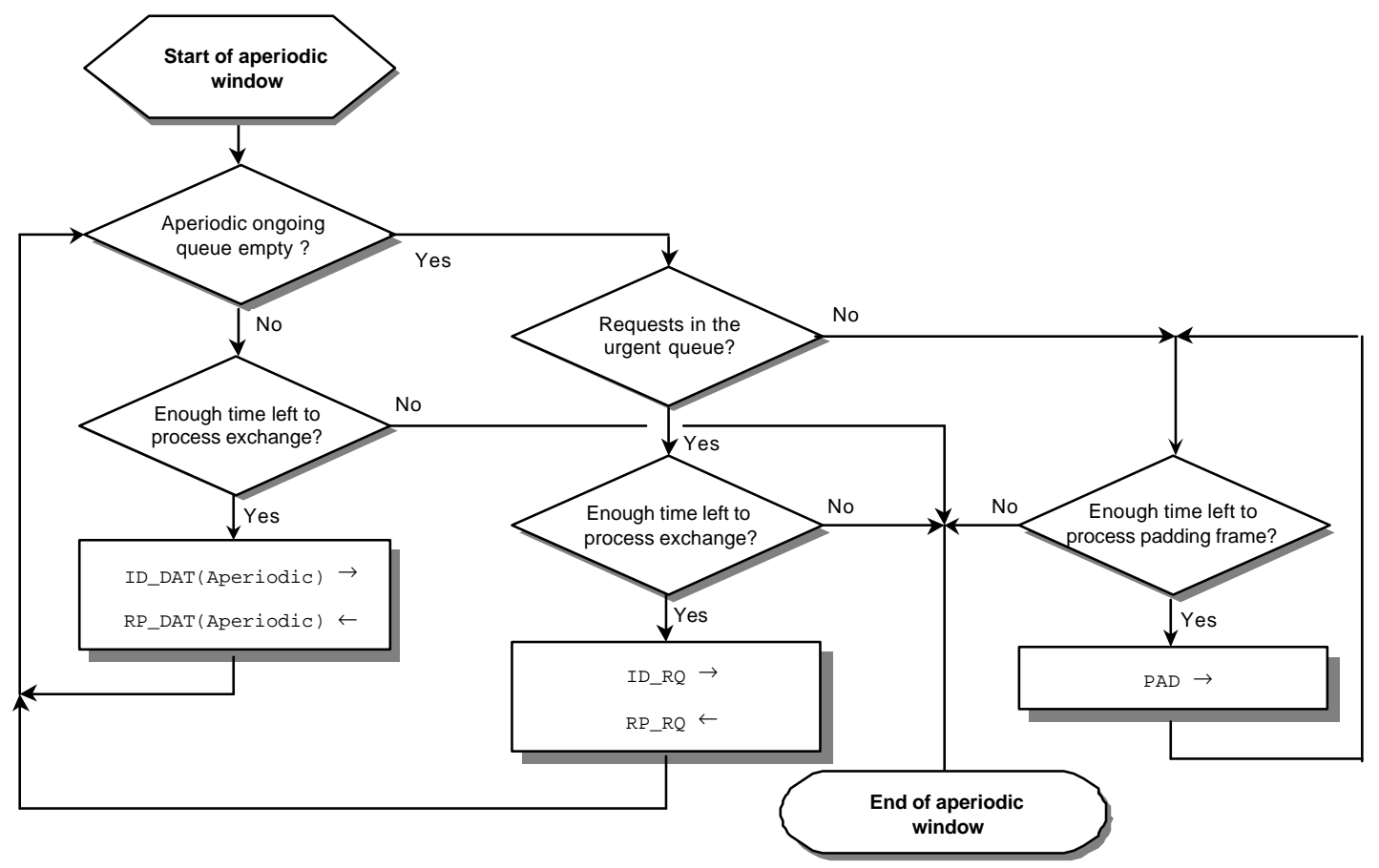

Fig. 5. This figure illustrates the (urgent) aperiodic buffer transfer mechanisms of the WorldFIP protocol

\section{Response Time Analysis of WorldFIP Traffic}

\subsection{Model for the WorldFIP Buffer Transfers}

Consider the following model for the WorldFIP buffer transfers (periodic and aperiodic). Assume a system with $n p$ periodic variables $\left(V p_{i}, i=1, \ldots, n p\right)$ and $n a$ aperiodic variables $\left(V a_{j}, j=1, \ldots, n a\right)$. Each periodic variable is characterised as:

$$
V p_{i}=\left(T p_{i}, C p_{i}\right)
$$

where $T p_{i}$ corresponds to the periodicity of $V p_{i}$ and $C p_{i}$ is the length of the transaction corresponding to the buffer transfer of $V p_{i}$ (as given by equation (1)). Each aperiodic variable $V a_{j}$ is characterised as:

$$
V a_{j}=\left(T a_{j}\right)
$$

where $T a_{j}$ corresponds to the minimum inter-arrival time between two consecutive requests of $V a_{j}$.

\subsection{Building the WorldFIP BAT}

The real-time requirements of the WorldFIP periodic traffic can be easily guaranteed since the BAT implements a static schedule of the periodic buffer transfers. In this section we present three different algorithms to build the BAT schedule: two adapted algorithms from the well-known Rate Monotonic (RM) and Earliest Deadline First (EDF) algorithms [14], respectively, and also a third algorithm which we denote as the Deferred Release (DR) algorithm.

Building the BAT schedule with either the RM or the EDF algorithms has several advantages, since it allows for the use of well-known schedulability analysis theory. 
Considering the RM scheduling algorithm, variables are scheduled according to their periodicity: the variable with the smallest periodicity will have the highest priority. If several variables have the same periodicity (priority tie), the higher priority is given to the variable with the smaller identifier. In appendix A.1, a detailed algorithm for building the BAT using the RM algorithm is presented. The algorithm indicates whether all traffic is schedulable or not (line 22). In the algorithm, the vector load [ ] is used to store the load in each micro-cycle as the traffic is scheduled. It also assumes that the array $V p[$,$] is ordered from the variable with the shortest period$ $(V p[1]$,$) to the variable with the longest period (V p[n p]$,$) . The RM approach for building the WorldFIP BAT$ has some aspects that are worthwhile to be addressed. The following example highlights some of these aspects.

Consider the set of periodic buffer transfers presented in Table 1, where the network data rate is $1 \mathrm{Mbps}$ instead of $2.5 \mathrm{Mbps}$, which results in longer messages: $C p_{i}=(64+80) / 1+2 \times 20=184 \mu \mathrm{s}$ (Table 2$)$. Note that each micro-cycle is able to schedule up to 5 periodic buffer transfers (Fig. 6). As a consequence, there is the insertion of idle time in the resulting schedule, since the remaining time in the first micro-cycle $(1 \mathrm{~ms}-5 \times 184 \mu \mathrm{s}=80 \mu \mathrm{s})$ is not enough to process a sixth transaction.

Table 2: BAT (using RM) for example of Table 1, considering $C p_{i}=184 \mu \mathrm{s}$

\begin{tabular}{c|cccccccccccc} 
& \multicolumn{11}{c}{ Micro-cycle } \\
\cline { 2 - 12 } & $\mathbf{1}$ & $\mathbf{2}$ & $\mathbf{3}$ & $\mathbf{4}$ & $\mathbf{5}$ & $\mathbf{6}$ & $\mathbf{7}$ & $\mathbf{8}$ & $\mathbf{9}$ & $\mathbf{1 0}$ & $\mathbf{1 1}$ & $\mathbf{1 2}$ \\
\hline bat $[A$, cycle $]$ & 1 & 1 & 1 & 1 & 1 & 1 & 1 & 1 & 1 & 1 & 1 & 1 \\
bat[B,cycle $]$ & 1 & 0 & 1 & 0 & 1 & 0 & 1 & 0 & 1 & 0 & 1 & 0 \\
bat[C,cycle $]$ & 1 & 0 & 0 & 1 & 0 & 0 & 1 & 0 & 0 & 1 & 0 & 0 \\
bat $[$ D,cycle $]$ & 1 & 0 & 0 & 0 & 1 & 0 & 0 & 0 & 1 & 0 & 0 & 0 \\
bat $[$ E,cycle $]$ & 1 & 0 & 0 & 0 & 1 & 0 & 0 & 0 & 1 & 0 & 0 & 0 \\
bat $[F$, cycle $]$ & 0 & 1 & 0 & 0 & 0 & 0 & 1 & 0 & 0 & 0 & 0 & 0
\end{tabular}

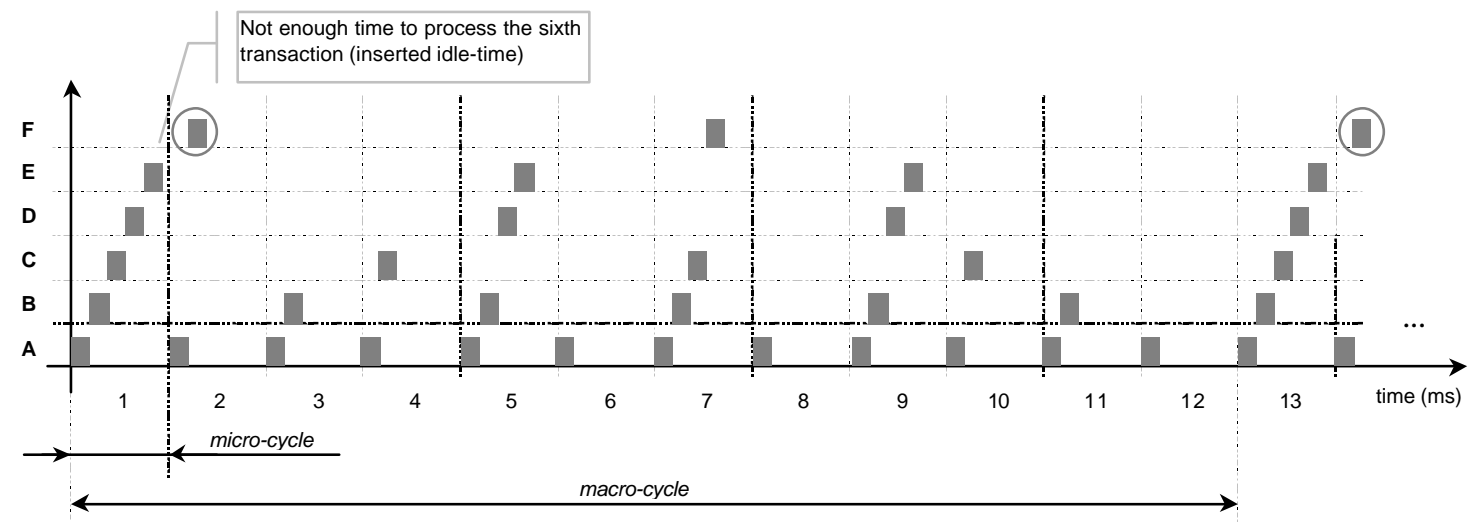

Fig. 6. Schedule (RM approach) for the set example of Table 2

An alternative to the use of the RM algorithm to build the BAT schedule is the EDF algorithm. When compared to the RM scheduling, one of the known advantages of the EDF scheduling is that it allows a better utilisation [14]. Considering the EDF algorithm, variables are scheduled according to the earliest deadline (in terms of micro-cycle). If several variables have the same deadline (priority tie), the higher priority is given to the variable with the earliest request.

In appendix A.2, a detailed algorithm for building the BAT with the EDF algorithm is presented. In the algorithm, the array $\operatorname{disp}[$,$] is used to store in \operatorname{disp}[i, 1]$ whether there is any pending request for variable $i$, in $\operatorname{disp}[i, 2]$ the related deadline (multiple of the micro-cycle), and in disp $[i, 3]$ the micro-cycle at which the request is made.

The increased utilisation allowed by the EDF scheduling algorithm is emphasised, considering the set of periodic buffer transfers shown in Table 3 (utilisation equal to 90\%). 
Table 3: Exa mple set of periodic buffer transfers $(C p=0.30 \mathrm{~ms})$

\begin{tabular}{l|cccccc} 
Identifier & A & B & C & D & E & F \\
\hline Periodicity (ms) & 1 & 2 & 2 & 3 & 3 & 3
\end{tabular}

Considering the RM algorithm, the first request for $V p_{F}$ would miss its deadline (Table 4). In fact, there is no empty slot for variable $V p_{F}$ in the first 3 micro-cycles, since each micro-cycle is only able to schedule up to 3 buffer transfers.

Table 4: BAT (using RM) for example of Table 3

\begin{tabular}{c|cccccc} 
& \multicolumn{7}{|c}{ Micro-cycle } \\
\cline { 2 - 7 } & $\mathbf{1}$ & $\mathbf{2}$ & $\mathbf{3}$ & $\mathbf{4}$ & $\mathbf{5}$ & $\mathbf{6}$ \\
\hline bat[,cycle $]$ & 1 & 1 & 1 & 1 & 1 & 1 \\
bat[B,cycle $]$ & 1 & 0 & 1 & 0 & 1 & 0 \\
bat[C,cycle $]$ & 1 & 0 & 1 & 0 & 1 & 0 \\
ba[D,cycle $]$ & 0 & 1 & 0 & 1 & 0 & 0 \\
ba[[E,cycle $]$ & 0 & 1 & 0 & 1 & 0 & 0 \\
bat[F,cycle $]$ & $\mathrm{X}$ & 0 & 0 & 0 & 0 & 1
\end{tabular}

Considering the EDF algorithm, the first request for $V p_{F}$ does not miss its deadline (Table 5), since the second request of variable $V p_{C}$ is scheduled only in the fourth micro-cycle. Thus, by increasing the communication jitter, the set of periodic buffer transfers shown in Table 3 is now schedulable.

Table 5: BAT (using EDF) for example of Table 3

\begin{tabular}{c|cccccc} 
& \multicolumn{7}{|c}{ Micro-cycle } \\
\cline { 2 - 7 } & $\mathbf{1}$ & $\mathbf{2}$ & $\mathbf{3}$ & $\mathbf{4}$ & $\mathbf{5}$ & $\mathbf{6}$ \\
\hline ba[A,cycle $]$ & 1 & 1 & 1 & 1 & 1 & 1 \\
bat[B,cycle $]$ & 1 & 0 & 1 & 0 & 1 & 0 \\
ba[C,cycle $]$ & 1 & 0 & 0 & 1 & 1 & 0 \\
bat[D,cycle $]$ & 0 & 1 & 0 & 1 & 0 & 0 \\
bat[E,cycle $]$ & 0 & 1 & 0 & 0 & 0 & 1 \\
bat[F,cycle $]$ & 0 & 0 & 1 & 0 & 0 & 1
\end{tabular}

An particular characteristic of using EDF to build the WorldFIP BAT is that, in spite of having an utilisation smaller than $100 \%$, there is not any available time left on the BAT to schedule another aperiodic buffer transfer. This is due to the inserted idle-time in each micro-cycle, which has the length $1000-3 \times 300=100 \mu$ s.

With RM and EDF algorithms, besides the inserted idle time aspect, variables that are scheduled in subsequent micro-cycles will suffer from an increased communication jitter. For instance, the reduction of the jitter associated to the transfer of the low priority variables can be easily achieved by manipulation of the release time of such variables.

In appendix A.3, a detailed algorithm for building the BAT using a Deferred Release (DR) approach is presented. In this algorithm, each variable is schedule according to its exact periodicity (in terms of number of micro-cycles). That is, either the variable is scheduled in the correct micro-cycle, or it is not scheduled (line 25). The release time of each periodic variable is chosen in order to minimise the maximum length of the periodic windows in the resulting BAT schedule (line 18).

For instance, the resulting schedule for the example of Table $1\left(C p_{i}=184 \mu \mathrm{s}\right)$ would be as presented in Table 6 and Fig. 7 (compared to Table 2 and Fig. 6 for the RM case, results on a clear reduction of the communication jitter): 
Table 6: BAT (using DR) for example of Table $1\left(C p_{i}=184 \mu \mathrm{s}\right)$

\begin{tabular}{c|cccccccccccc} 
& \multicolumn{11}{c}{} & \multicolumn{11}{c}{ Micro-cycle } \\
\cline { 2 - 14 } & $\mathbf{1}$ & $\mathbf{2}$ & $\mathbf{3}$ & $\mathbf{4}$ & $\mathbf{5}$ & $\mathbf{6}$ & $\mathbf{7}$ & $\mathbf{8}$ & $\mathbf{9}$ & $\mathbf{1 0}$ & $\mathbf{1 1}$ & $\mathbf{1 2}$ \\
\hline bat $[$ A,cycle $]$ & 1 & 1 & 1 & 1 & 1 & 1 & 1 & 1 & 1 & 1 & 1 & 1 \\
bat $[$ B,cycle $]$ & 1 & 0 & 1 & 0 & 1 & 0 & 1 & 0 & 1 & 0 & 1 & 0 \\
bat $[$ C,cycle $]$ & 1 & 0 & 0 & 1 & 0 & 0 & 1 & 0 & 0 & 1 & 0 & 0 \\
bat $[D$, cycle $]$ & 0 & 1 & 0 & 0 & 0 & 1 & 0 & 0 & 0 & 1 & 0 & 0 \\
bat $[$ E,cycle $]$ & 0 & 0 & 0 & 1 & 0 & 0 & 0 & 1 & 0 & 0 & 0 & 1 \\
bat $[F$, cycle $]$ & 0 & 1 & 0 & 0 & 0 & 0 & 0 & 1 & 0 & 0 & 0 & 0
\end{tabular}

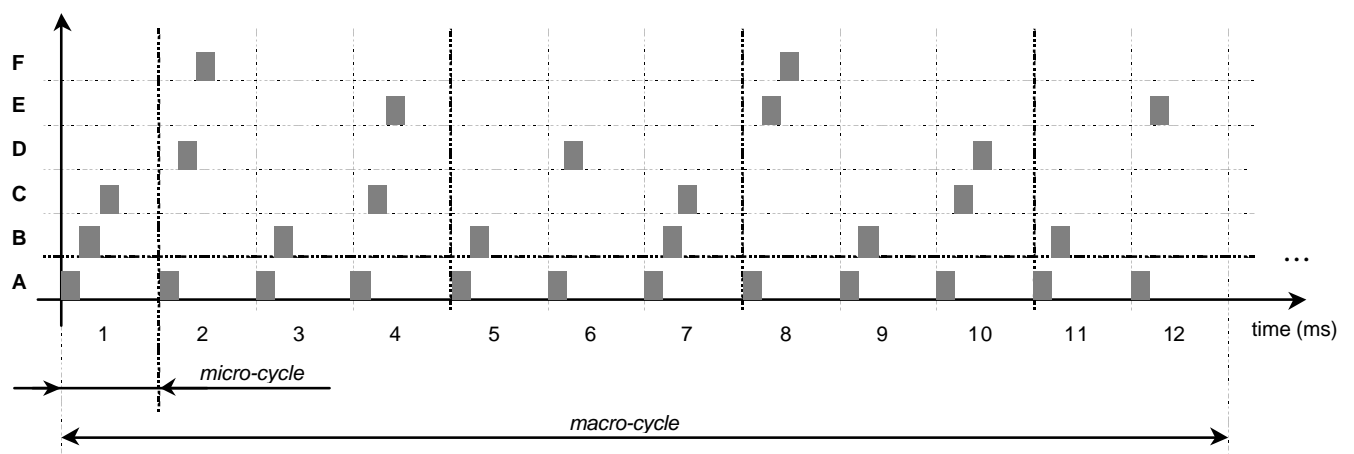

Fig. 7. Schedule (DR approach) for the set example of Table 6

\subsection{Response Time Characterisation of an Aperiodic Buffer Transfer}

The response time of an aperiodic buffer transfer is function of the periodic traffic pattern, since the length of each aperiodic window is function of the generated BAT schedule. Thus, the exact characterisation of the periodic traffic pattern (as defined in the BAT schedule) is fundamental for the response time analysis of the aperiodic traffic.

We define the response time of an aperiodic buffer transfer as the time interval between placing, at time instant $t_{0}$, the request in the local urgent queue and the completion of the buffer transfer concerning the aperiodic variable $V a_{j}$ in a BA's aperiodic window (Fig. 4). This response time includes the following three components:

1. the time elapsed between $t_{0}$ and the time instant when the requesting station (station $k$ ) is able to indicate the BA (via an RP_DAT frame, with the request bit set) that there is a pending aperiodic request. We define this time interval as the dead interval of a producer station (at the end of this interval, the BA is aware of a pending request in station $k$, without being able to identify it);

2. the time interval during which the request indication stays in the BA's urgent queue till the related $I D \_R Q / R P \_R Q$ pair of frames is processed in an aperiodic window (at the end of this interval, the BA knows which is the pending request in station $k$ );

3. the time interval during which the request for variable $V a_{j}$ stays in the BA's ongoing aperiodic queue till the related ID_DAT_Ap / RP_DAT pair of frames is processed in an aperiodic window.

These two last components (2 and 3) can be grouped as the BA's processing interval of an aperiodic buffer transfer. All these components must be upper bounded, in order to have a bounded response time for an aperiodic buffer transfer. 


\subsection{Upper Bound for the Dead Interval}

The upper bound for the dead interval in a station $k$ is related to the smallest scanning period of a periodic variable, produced in that station. It is important to note that a periodic variable $\left(V p_{i}\right)$ is not polled at regular intervals due to the communication jitter inherent to the BAT schedule. Therefore, the upper bound for the dead interval in a station $k$ is:

$$
\sigma^{k}=T p_{j}+J_{V p_{j}}+C p_{j} \text {, with } V p_{j}: T p_{j}=\min _{V p_{i} \text { producedin } k}\left\{T p_{i}\right\}
$$

where $J_{V p_{j}}$ is the maximum communication jitter of that $V p_{j}$. We consider that a local aperiodic request is processed (setting the request bit in the $R P \_D A T$ frame) only if it arrives before the start of the related ID_DAT . Hence, the term $C p_{j}$ is included in equation (4).

For instance, concerning the set of periodic variables of Table 1, if variable $F$ is the only produced variable at station $k$, then $\sigma^{k}=6+0.20+0.098=6.30 \mathrm{~ms}$ (see Section 2.3 for the evaluation of the jitter).

In appendix A.4 we describe a detailed algorithm for the evaluation of the communication jitter associated to a periodic variable, which performs the analysis of the generated BAT schedule. This algorithm is the basis for the evaluation of the dead interval in a specific station $k$.

\subsection{Upper Bound for the Processing Interval}

We assume that all the aperiodic traffic has a minimum inter-arrival time between requests, which is greater than its worst-case response time. Therefore, no aperiodic request appears before the completion of a previous one. Hence, the maximum number of aperiodic requests pending in the $\mathrm{BA}$ is $n a$, with $n a$ being the number of aperiodic variables in the network (Section 3.1).

The upper bound for the BA's processing interval occurs when both the periodic and aperiodic traffic are simultaneously at their maximum load.

We consider that for each aperiodic variable a request for identification must be made, and thus the network load for the aperiodic traffic is maximised. As a consequence, to process the maximum number of aperiodic variables, the aperiodic windows will perform alternately sequences of ( $\left.I D \_R Q / R P \_R Q\right)$ and (ID_DAT / $R P \_D A T$ ), as the BA gives priority to the ongoing aperiodic queue (see Fig. 5). That is, the number of transactions to be processed is $2 \times n a$.

If all the aperiodic variables have a similar length, the length of the longest transaction in an aperiodic window may be defined as:

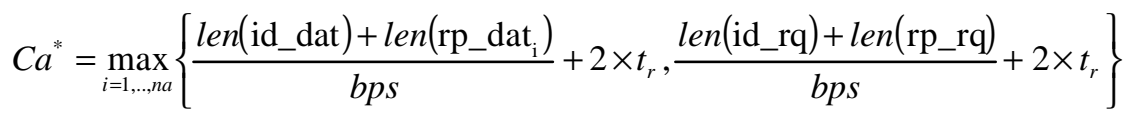

We define the BA's processing interval of na consecutive aperiodic requests, as an aperiodic busy interval (ABI), since all aperiodic windows within this interval are used to process aperiodic traffic. We denote $N_{A B I}(j)$ as the number of micro-cycles during an ABI that starts at micro-cycle $j$ and len_abi $(j)$ as the length of such ABI.

The upper bound for the length of the ABI occurs when the ABI is starting at the critical micro-cycle, which is defined as the micro-cycle that leads to the longest ABI.

It is obvious that using either the RM or the EDF scheduling policies to build the BAT, the critical microcycle is the first one of the macro-cycle. However for the case of different approaches to build the BAT schedule (e.g. the presented DR algorithm), the critical micro-cycle may not be the first one. For these other scheduling 
algorithms, it is necessary to determine which is the critical micro-cycle, in order to evaluate the length of the longest aperiodic busy interval.

The length of the longest ABI is evaluated as follows. Firstly, we determine recursively the value for each $N_{A B I}(\mathrm{j})$. After, we evaluate the length of each ABI $\left(l e n \_a b i(j)\right)$, considering that during one ABI there are only $\left(N_{A B I}(j)-1\right)$ micro-cycles which are completely utilised.

The length of the aperiodic window in the $l^{t h}$ cycle $(l=1, . ., N, N+1, .$.$) is:$

$$
a w(l)=\mu C y-\sum_{i=1}^{n p}\left(b a t[i, l *] \times C p_{i}\right)
$$

where $b a t\left[i, l^{*}\right]$ is a matrix representing the schedule of the periodic traffic and $l^{*}=[(l-1) \bmod N]+1$. The use of variable $l^{*}$ stresses that cycle $l=1$ is equivalent to cycle $l=N+1$, as a macro-cycle has only $N$ micro-cycles. Therefore, the number of aperiodic transactions that fit in the $l^{\text {th }}$ aperiodic window is:

$$
\operatorname{nap}(l)=\left\lfloor\frac{a w(l *)}{C a *}\right\rfloor
$$

The number of micro-cycles during an ABI starting at micro-cycle $j\left(N_{A B I}(j)\right)$ is then:

$$
N_{A B I}(j)=\min \{\Psi\}, \quad \text { with } \Psi=\sum_{l=j}^{(j-1)+N_{A B I}} n a p(l *) \wedge \Psi \geq 2 \times n a
$$

This value must be recursively evaluated for each micro-cycle $j$, since the number of aperiodic transactions that fit in an aperiodic window depends on the micro-cycle that is being considered. The recursion stops when the number of available "slots" (each "slot" with the length of $\mathrm{Ca}^{*}$ ) is at least $2 \times n a$.

Knowing the number of micro-cycles during an ABI, the length of each aperiodic busy interval (len_abi(j)) may be evaluated as follows:

$$
l e n \_a b i(j)=\left(N_{A B I}(j)-1\right) \times \mu C y+\sum_{i=1}^{n p}\left(b a t\left[i,\left((j-1)+N_{A B I}(j)\right) *\right] \times C p_{i}\right)+\left(2 \times n a-\sum_{l=j}^{(j-1)+N_{A B I}(j)-1} n a p(l *)\right) \times C a^{*}
$$

where $\left(N_{A B I}(j)-1\right) \times \mu C y$ indicates the length of the completely utilised micro-cycles, $\sum_{i=1, . ., n p}\left(\right.$ bat $\left.\left[i,\left(j-1+\mathrm{N}_{\mathrm{ABI}}(j)\right)^{*}\right] \times C p_{i}\right)$ indicates the length of the periodic window in the last micro-cycle of the ABI, with $\left.\left((j-1)+\mathrm{N}_{\mathrm{ABI}}(j)\right)^{*}=\left[(j-1)+\mathrm{N}_{\mathrm{ABI}}(j)-1\right) \bmod N\right]+1$ and $\left(2 \times n a-\sum_{l=1, \ldots . j+N(j)-2} n a p(l *)\right) \times C a^{*}$ indicates how many aperiodic transactions are still pending to be processed on the last micro-cycle of the ABI.

The length of the longest $\mathrm{ABI}$ is then:

$$
l e n_{-} a b i=\max _{j \in\{1, \ldots, N\}}\left\{l e n_{-} a b i(j)\right\}
$$

In appendix A.5 a detailed algorithm for evaluating the length of the longest ABI is provided.

\subsection{Worst-Case Response Time for the Aperiodic Requests}

The worst-case response time of an aperiodic buffer transfer at station $k$ is equal to the length of the dead interval of that station plus the length of the longest $\mathrm{ABI}$ :

$$
R a^{k}=\sigma^{k}+l e n_{-} a b i
$$

Thus, the minimum inter-arrival time between any two consecutive aperiodic requests of the same aperiodic variable in a station $k$ is: 


$$
T a_{j} \geq R a^{k}=\sigma^{k}+l e n_{-} a b i
$$

Inequality (12) presents a pre-run-time schedulability test, which can be used to validate the real-time requirements of WorldFIP applications.

\section{Numerical Example}

Consider that a WorldFIP network with 6 periodic variables (as defined in Table 7) must also support 7 aperiodic buffer exchanges. Assume that the length of the each $V p_{i}, \forall_{i}$ is $C p_{i}=C p=200 \mu \mathrm{s}$, and that the length of an aperiodic buffer transfer is $C a^{*}=100 \mu \mathrm{s}$. The value of the micro-cycle is $1 \mathrm{~ms}$.

Table 7: Example Set of Periodic Buffer Transfers

\begin{tabular}{l|cccccc} 
Identifier & A & B & C & D & E & F \\
\hline Periodicity (ms) & 1 & 2 & 2 & 3 & 3 & 6
\end{tabular}

If the BAT is implemented as shown in Table 8 (enforcing micro-cycles 4 and 6 to be the most loaded ones), the longest ABI will not be at the beginning of the macro-cycle. Thus, the worst-case response time for an aperiodic transfer will occur when the request arrives to the BA at the beginning of the longest $\mathrm{ABI}$ and not at the beginning of the macro-cycle.

Table 8: BAT for the numerical example

\begin{tabular}{c|cccccc} 
& \multicolumn{7}{|c}{ Micro-cycle } \\
\cline { 2 - 7 } & $\mathbf{1}$ & $\mathbf{2}$ & $\mathbf{3}$ & $\mathbf{4}$ & $\mathbf{5}$ & $\mathbf{6}$ \\
\hline ba[A,cycle $]$ & 1 & 1 & 1 & 1 & 1 & 1 \\
ba $[$ B,cycle $]$ & 0 & 1 & 0 & 1 & 0 & 1 \\
ba [C,cycle $]$ & 0 & 1 & 0 & 1 & 0 & 1 \\
ba[D,cycle $]$ & 1 & 0 & 0 & 1 & 0 & 0 \\
bat[E,cycle $]$ & 0 & 0 & 1 & 0 & 0 & 1 \\
bat[F,cycle $]$ & 0 & 0 & 1 & 0 & 0 & 0
\end{tabular}

To evaluate the worst-case response time of an aperiodic transfer, we will proceed as defined in Section 3.5. The length of each aperiodic window during the macro-cycle (6) and the number of aperiodic transactions that fit in each aperiodic window (7) are, respectively (Table 9):

Table 9: Aperiodic windows for example of Table 8

\begin{tabular}{c|cccccc} 
Micro-cycles & $\mathbf{1}$ & $\mathbf{2}$ & $\mathbf{3}$ & $\mathbf{4}$ & $\mathbf{5}$ & $\mathbf{6}$ \\
\hline $\operatorname{aw}(l)(\mu \mathrm{s})$ & 600 & 400 & 400 & 200 & 800 & 200 \\
$\operatorname{nap}(l)$ & 6 & 4 & 4 & 2 & 8 & 2
\end{tabular}

Using the recurrence relationship given by equation (8), we may now evaluate the number of micro-cycles during an $\mathrm{ABI}$, for each micro-cycle $j$. For the case of an $\mathrm{ABI}$ starting at the second micro-cycle $(j=2), N_{A B I}(2)$ is evaluated as follows:

$$
\begin{aligned}
& N_{A B I}(2)=1, \quad \Psi=\sum_{l=2}^{2} n a p(l *)=4<14 \\
& N_{A B I}(2)=2, \quad \Psi=\sum_{l=2}^{3} n a p(l *)=4+4<14
\end{aligned}
$$




$$
\begin{aligned}
& N_{A B I}(2)=3, \quad \Psi=\sum_{l=2}^{4} n a p(l *)=4+4+2<14 \\
& N_{A B I}(2)=4, \quad \Psi=\sum_{l=2}^{5} n a p(l *)=4+4+2+8 \geq 14, \text { and then iteration stops. Thus, } N_{A B I}(2)=4 .
\end{aligned}
$$

For the case of the ABIs starting in the other micro-cycles, the values for $N_{A B I}(j)$ are presented in Table 10. We may now evaluate the length of each ABI. For the case of an ABI starting at the second micro-cycle $(j=2)$, len_abi(2), the three components of equation (9) are evaluated as follows:

$$
\begin{aligned}
& \left(N_{A B I}(2)-1\right) \times \mu C y=3 \times 1000 \mu s=3000 \mu s \\
& \sum_{i=1}^{6}\left(\operatorname{bat}\left[i,\left(1+N_{A B I}(2)\right) *\right] \times C p_{i}\right)=\sum_{i=1}^{6}(\text { bat }[i, 5] \times 200 \mu s)=200 \mu s \\
& \left(2 \times n a-\sum_{l=2}^{4} n a p(l *)\right) \times 100 \mu s=(14-4-4-2) \times 100 \mu s=400 \mu s
\end{aligned}
$$

Thus, for the ABI starting at the second micro-cycle len_abi(2) $=3000+200+400=3600 \mu s$. For the case of the ABIs starting at the other micro-cycles, the values for len_abi(j) are also presented in Table 10.

Table 10: Characterisation of the aperiodic busy interval for each micro-cycle $j$

\begin{tabular}{c|cccccc} 
Micro-cycle $\boldsymbol{j}$ & $\mathbf{1}$ & $\mathbf{2}$ & $\mathbf{3}$ & $\mathbf{4}$ & $\mathbf{5}$ & $\mathbf{6}$ \\
\hline$N_{A B I}(j)$ & 3 & 4 & 3 & 4 & 3 & 4 \\
len_abi $(j)(\mathrm{ms})$ & 3.0 & 3.6 & 3.0 & 3.6 & 2.8 & 3.8
\end{tabular}

Thus, the length of the longest ABI is $3.8 \mathrm{~ms}$ and the critical micro-cycle is the sixth one. In Fig. 8, we illustrate the timing behaviour of an aperiodic buffer transfer for both the longest and the smallest ABIs, (AP_1) and (AP_2), respectively.

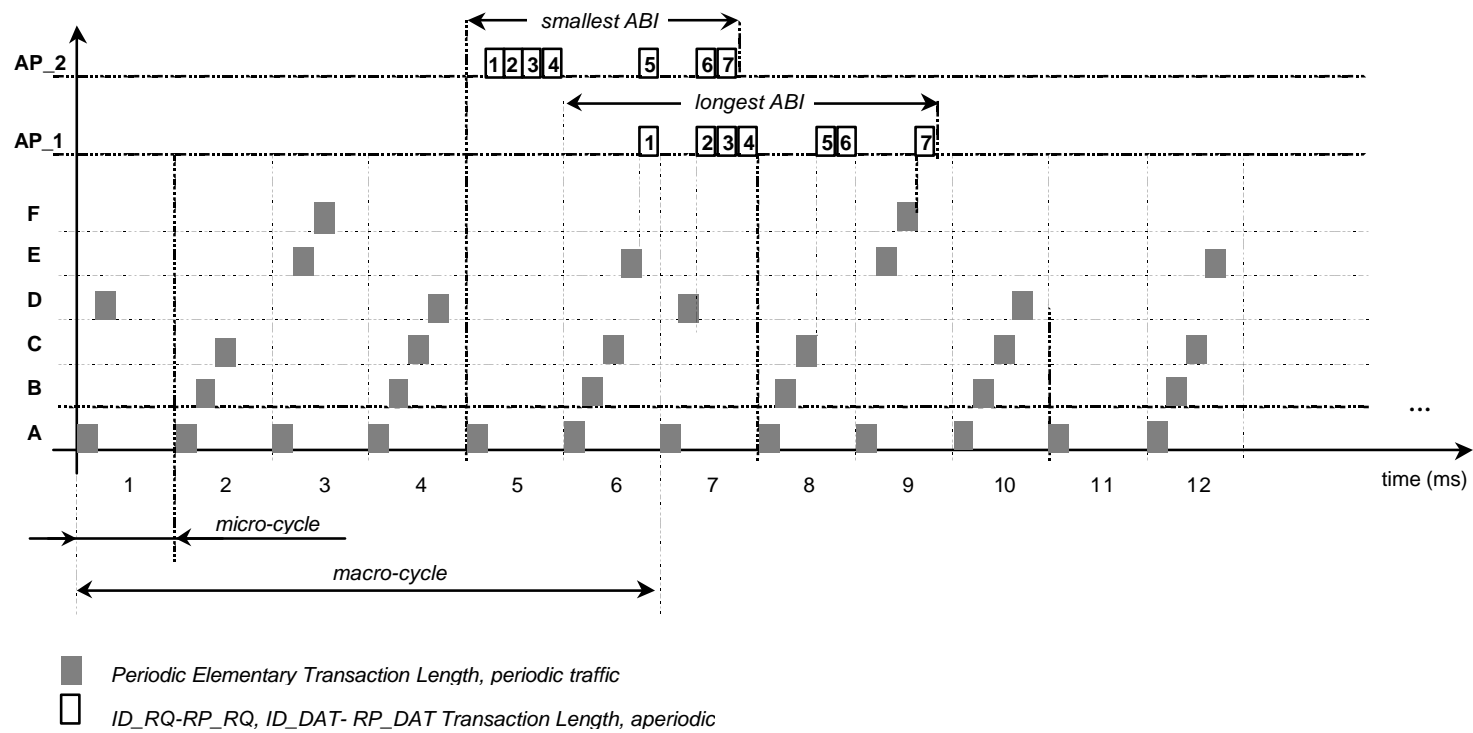

Fig. 8. This figure illustrates the timing behaviour of an aperiodic buffer transfer for the example of Table 10

Finally, to evaluate the worst-case response time of an aperiodic buffer transfer (11), we must consider the value of the dead interval. As an example, for a request made at a station $k$, which produces the periodic variable $F$ (assume that $F$ is the only periodic variable produced in station $k$ ), the dead interval (4) is $6.30 \mathrm{~ms}$. Therefore, the worst-case response time of an aperiodic variable requested in that station is $R a^{k}=6.30+3.80=10.10 \mathrm{~ms}$. 


\section{Conclusions}

The use of WorldFIP networks to support distributed computer-controlled systems (DCCS), requires the thorough analysis of the communication timing behaviour.

In this paper, we addressed the ability of the WorldFIP to cope with the real-time requirements of DCCS. The WorldFIP protocol is designed to support both periodic and aperiodic traffic. The real-time requirements for the periodic traffic can be easily guaranteed by an adequate setting of the WorldFIP BAT. However, for the aperiodic traffic more complex analysis must be made to evaluate the message's response time, in order to guarantee its real-time requirements. The main focus of this paper has been the analysis of the aperiodic message's response time.

To our best knowledge, few work has been done on the analysis of the message's response time in WorldFIP networks. Both 15] and [16] address the issue of finding worst-case response times for aperiodic requests in WorldFIP networks. However, those works are made under restrictive assumptions:

1. In [15] the authors consider that the aperiodic traffic is transferred only after all the periodic traffic in a macro-cycle, which leads to a very pessimistic result;

2. In [16] the authors consider equal lengths for the periodic windows, since they do not discuss the BAT construction and thus they cannot evaluate the length of the periodic window in each micro-cycle. Thus, the overall results are also very pessimistic.

More importantly, none of the analysis considered that at the end of each micro-cycle some transactions would simply not fit. They did also not take into account the communication jitter in the evaluation of the dead interval.

We significantly improved these results by approaching the analysis for the aperiodic traffic in an integrated manner with the methodologies used to build the BAT. Thus we reduced the pessimism considering the actual length of the periodic window in each micro-cycle. We also considered the effect of both the communication jitter and the inserted idle time.

\section{References}

[1] Groover, M. (1986). Automation Production Systems and Computer Integrated Manufacturing. PrenticeHall International Editions.

[2] Gutshke, W. and Mertins, K. (1987). CIM: Competitive Edge in Manufacturing. In Robotics and Computer Integrated Manufacturing, Vol. 2, No. 1, pp. 77-87.

[3] Pimentel, J. (1990). Communication Networks for Manufacturing. Prentice-Hall International Editions.

[4] Decotignie, J.-D. and Pleinevaux, P. (1993). A Survey on Industrial Communication Networks. In Annales des Télécomunications, Vol. 48, No. 9-10, pp. 55-63.

[5] Cardoso, A. and E. Tovar, 1996, Industrial Communication Networks: Issues on Heterogeneity and Internetworking, Proceedings of the $6^{\text {th }}$ International Conference on Flexible Automation and Intelligent Manufacturing (Begell House Publishers) 139-148.

[6] Cenelec, 1996, General Purpose Field Communication System. EN 50170, Vol. 1/3 (P-NET), Vol. 2/3 (PROFIBUS), Vol. $3 / 3$ (WorldFIP), Cenelec.

[7] P-Net, 1994, The P-NET Standard, (International P-NET User Organisation ApS).

[8] Profibus, 1992, PROFIBUS Standard DIN 19245 part I and II, Translated from German, (Profibus Nutzerorganisation e.V.).

[9] Afnor, 1990, Normes FIP NF C46-601 to NF C46-607, (UTE-Union Technique de l'Electricité).

[10] ISO 9506, 1990, Industrial Automation Systems - Manufacturing Message Specification (ISO). 
[11] Kim, Y., Jeong, S. and W. Kown, 1998, A Pre-Run-Time Scheduling Method for Distributed Real-Time Systems in a FIP Environment, Control Engineering Practice, Vol. 6, (Pergamon) 103-109.

[12] Kumaran, S. and J.-D. Decotignie, 1989, Multicycle Operations in a Field Bus: Application Layer Implications, Proceedings of IEEE Annual Conference of Industrial Electronics Society (IEEE) 531-536.

[13] Raja, P. and G. Noubir, 1993, Static and Dynamic Polling Mechanisms for Fieldbus Networks, Operating Systems Review, Vol. 27, No. 3, (ACM) 34-45.

[14] Liu, L. and J. Layland, 1973, Scheduling Algorithms for Multiprogramming in a Hard RealTime Environment, Journal of the ACM, 20(1), (ACM) 46-61.

[15] Vasques, F and G. Juanole (1994), Pre-run-time Schedulability Analysis in Fieldbus Networks, Proceedings of the 20th Annual Conference of the IEEE Industrial Electronics Society (IEEE) 1200-1204.

[16] Pedro, P. and A. Burns, 1997, Worst Case Response Time Analysis of Hard Real-time Sporadic Traffic in FIP Networks, Proceedings of the 9th Euromicro Workshop on Real-Time Systems (IEEE Computer Society Press) 3-10.

\section{Appendix}

\section{A.1. Rate Monotonic (RM) Algorithm for Building the BAT}

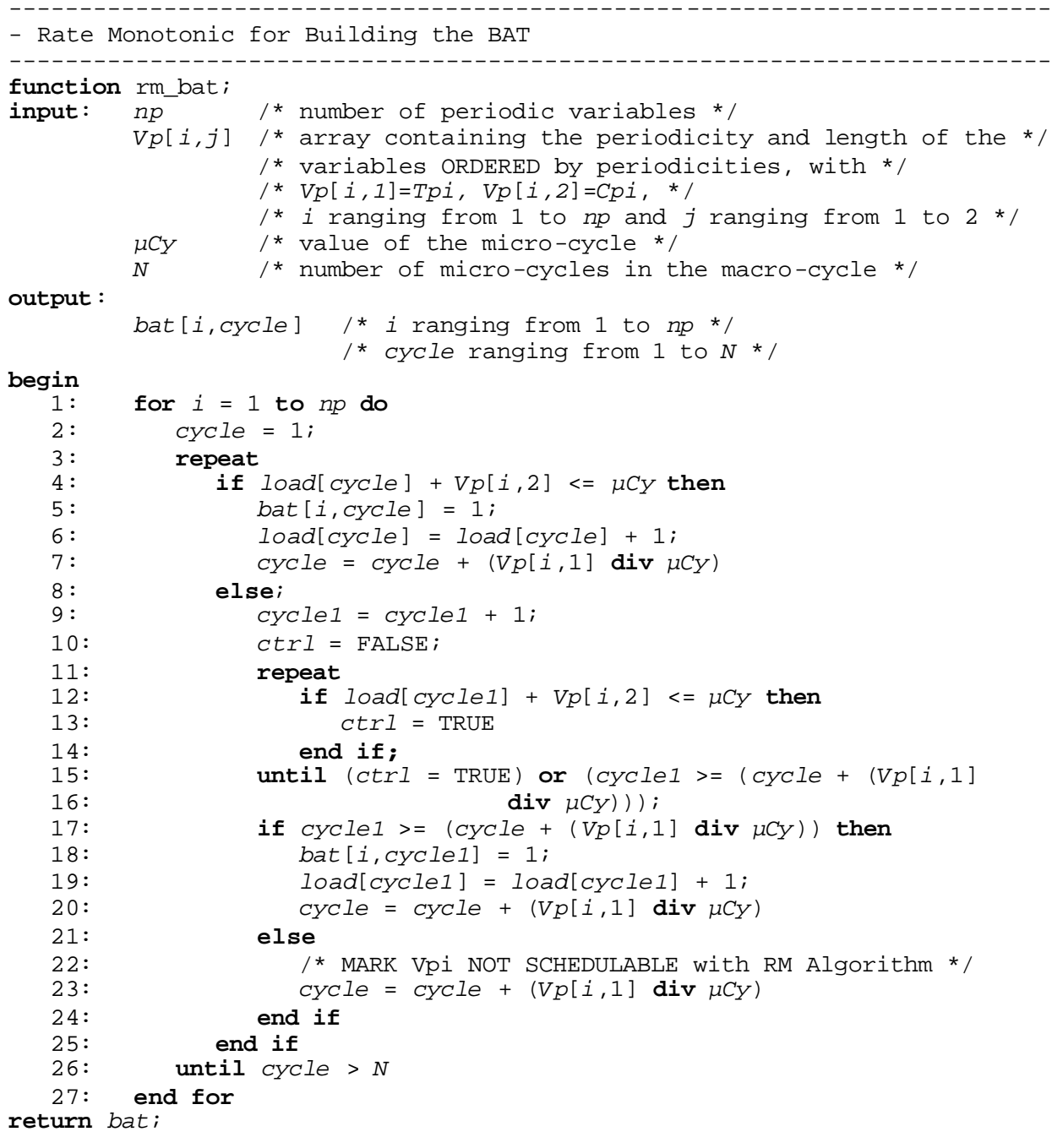




\section{A.2. Earliest Deadline (EDF) Algorithm for Building the BAT}

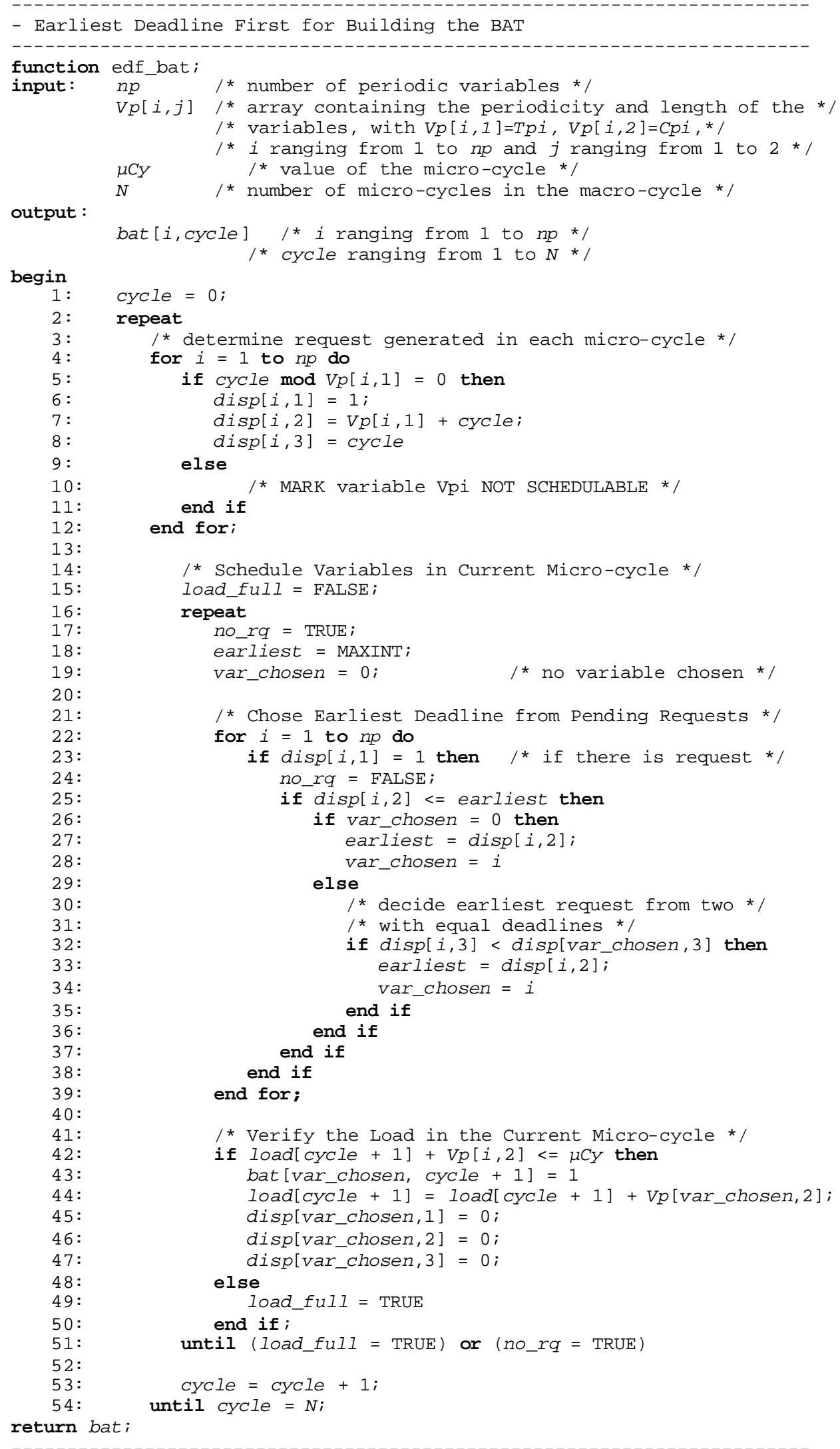




\section{A.3. Deferred Release (DR) Algorithm for Building the BAT}

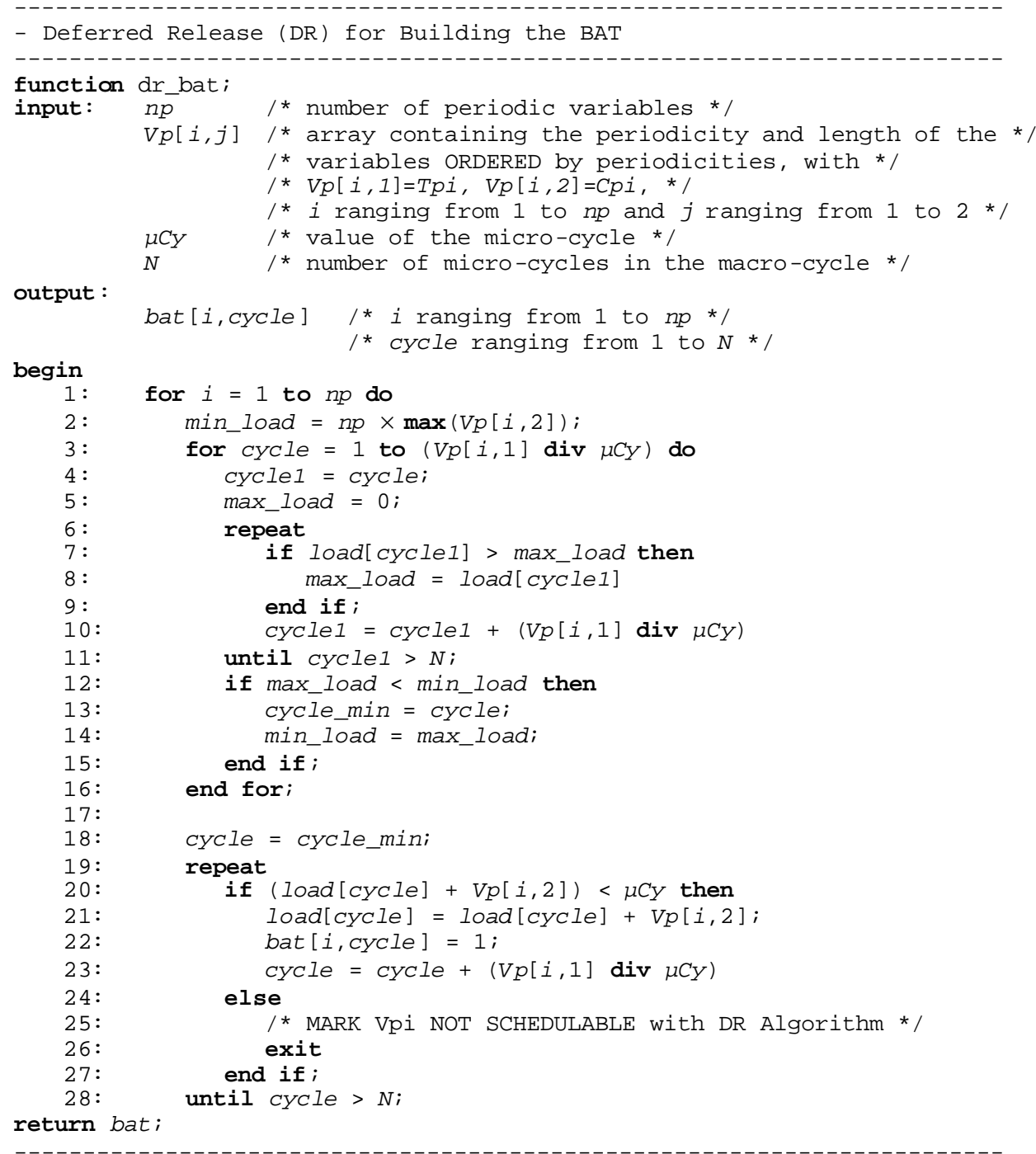

\section{A.4. Algorithm for the Evaluation of the Communication Jitter}

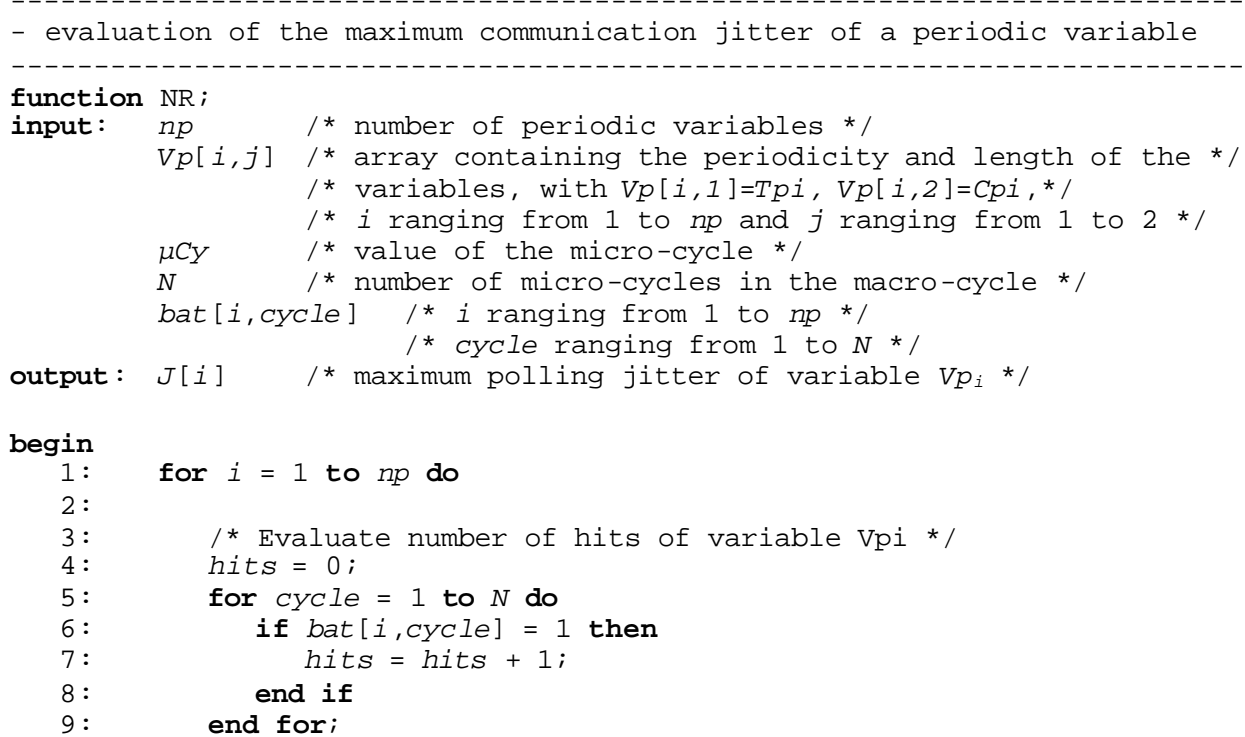




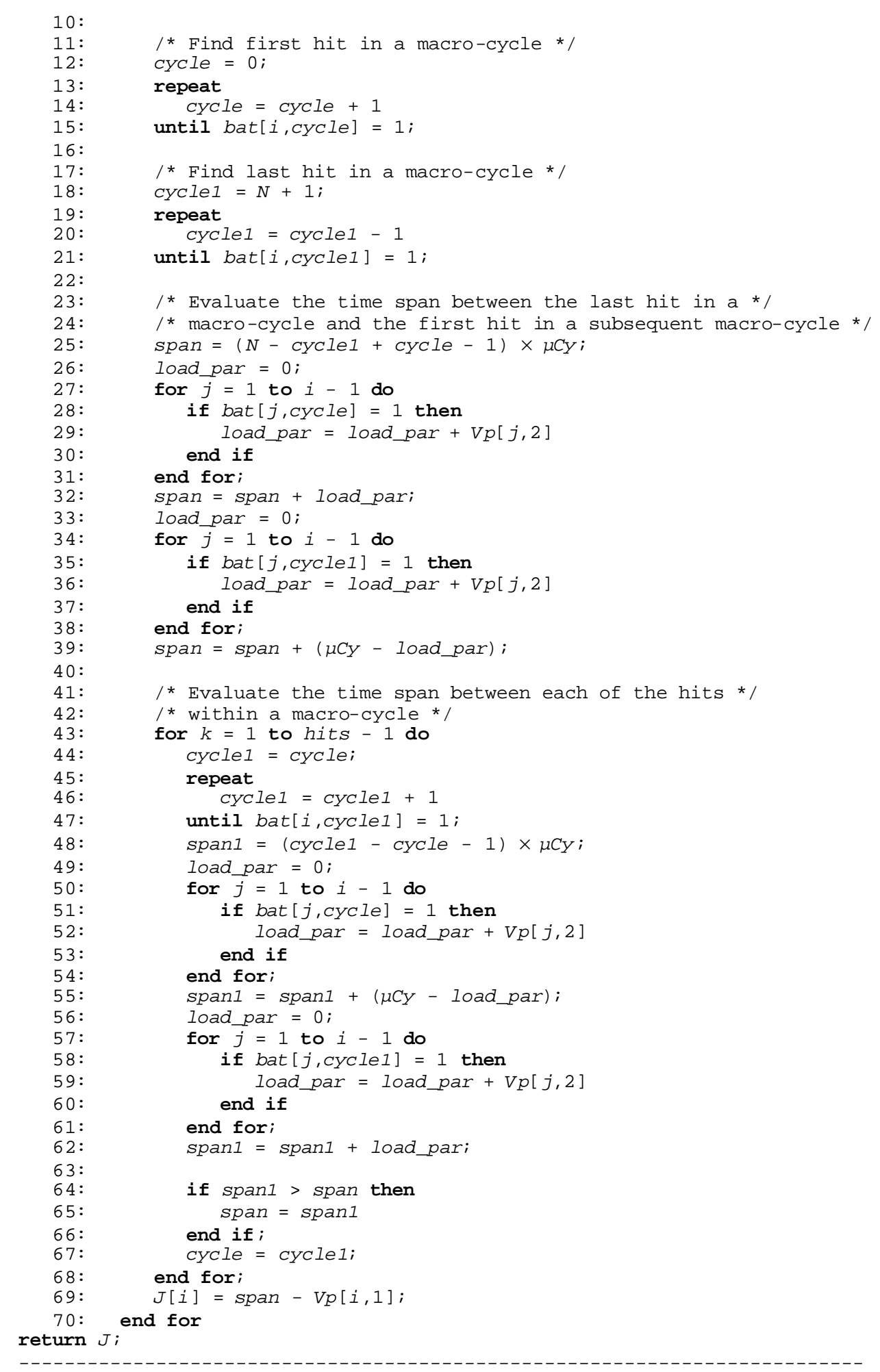

\section{A.5. Algorithm for the Evaluation of the Length of the ABI}

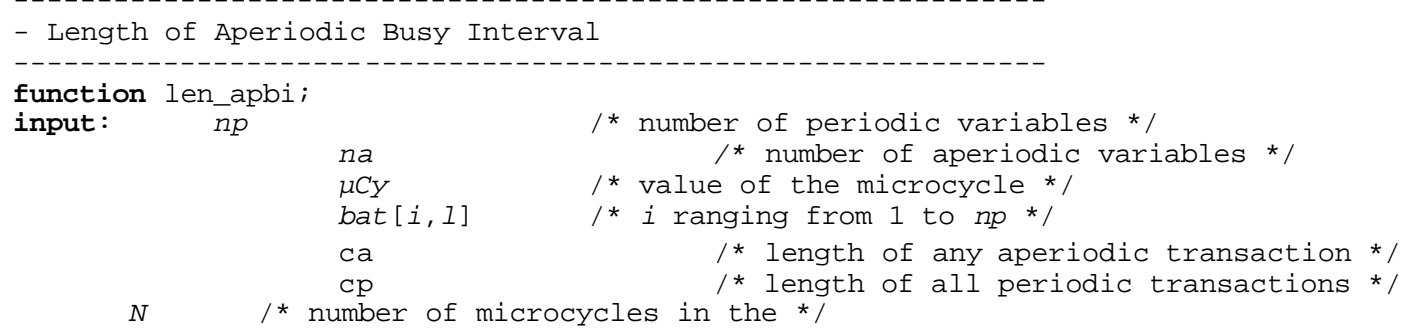




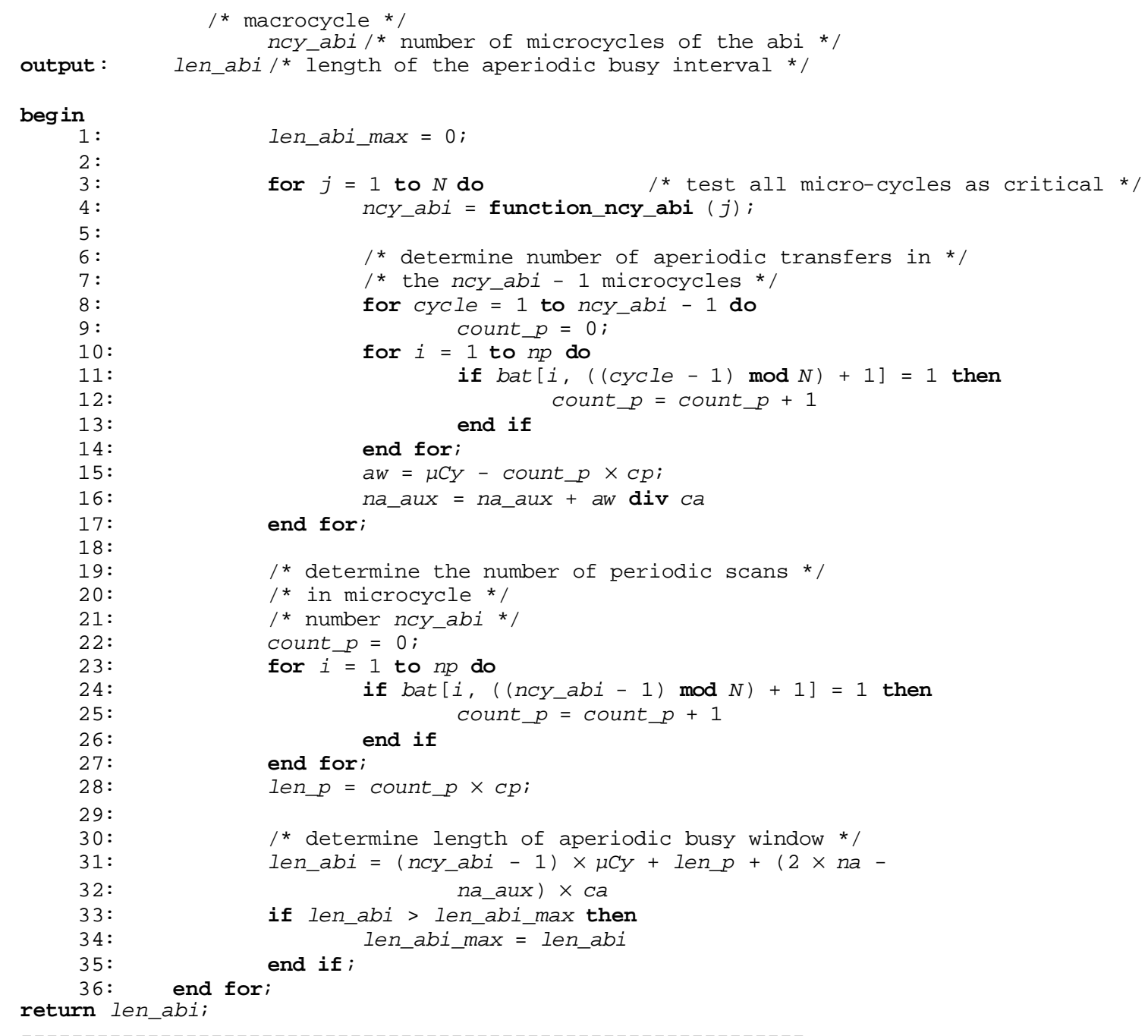

- Number of Cycles of the Aperiodic Busy Interval

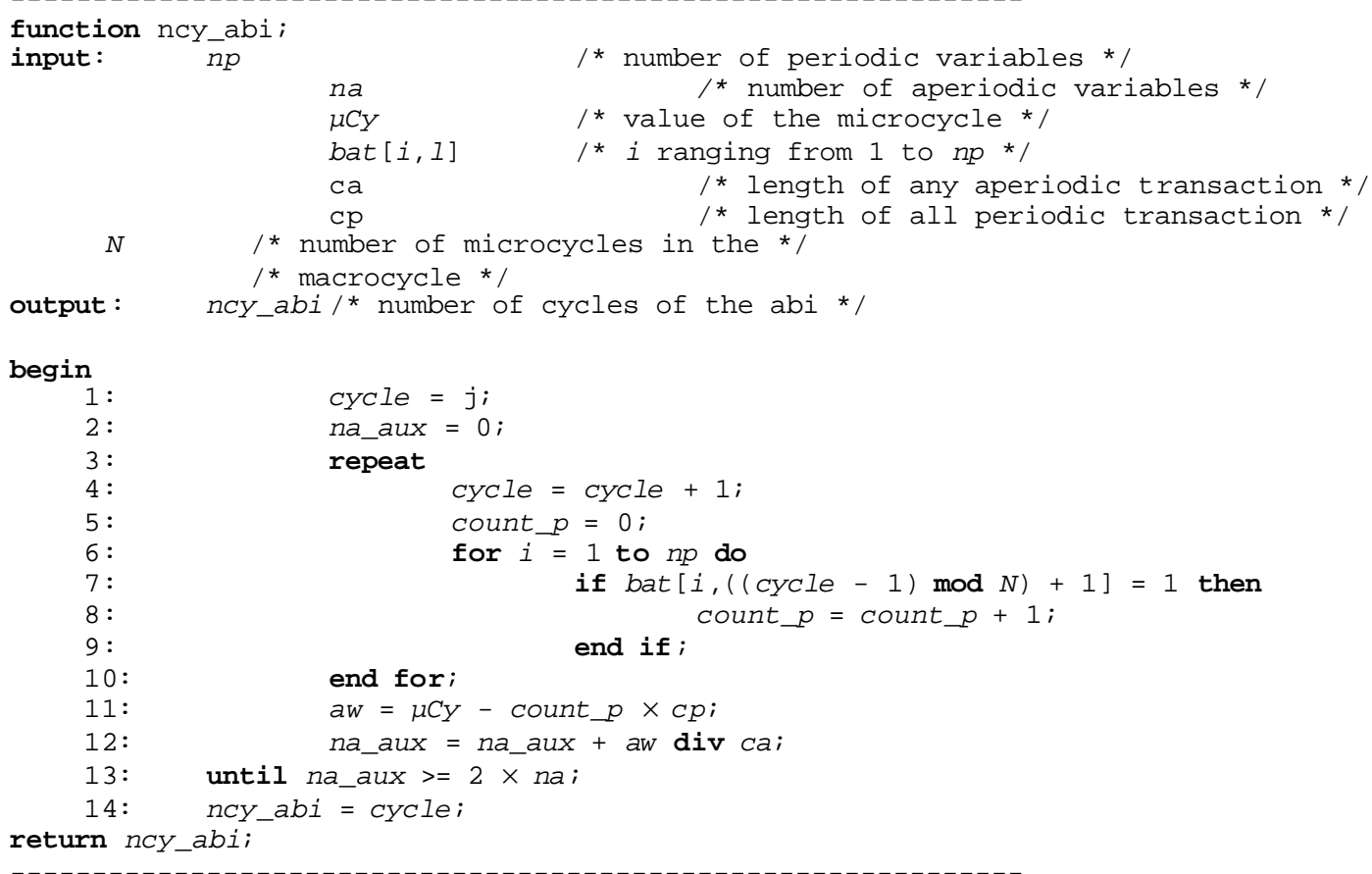

